vation a nuclear energy gap could be predicted as well as a "phase transition" at a critical angular momentum. This interesting phenomenon is strongly indicated in experiments on nuclei of high spin.

2) The adiabatic nature of the fission process has made it natural to consider the momentary energy as a function of deformations. Since much energy is absorbed by potential energy, the system is effectively at very low excitation in the barrier region. The adiabatic development of intrinsic states therefore governs the details of the fission process. A spectacular confirmation of this picture was found a few years ago with the discovery of fission isomers produced by a secondary barrier minimum. Even the rotational bands in this minimum have been seen. The modern understanding of fission derives directly from the discoveries in the 1950's.
3) The deformation of the self-consistent nuclear field leads in some cases to a radical reordering of the intrinsic states with deformation. In certain nuclear regions, these are so large that new shells occur for large deformations with magic numbers different from those of the shell model. Nuclear shapes can change drastically between nearby intrinsic states. These developments are closely linked to the spectacular success of the Nilsson model.

On closing this historical survey of the background to the 1975 Nobel prizes, I am struck by the following. It is quite possible to maintain the thesis that the initial theoretical discovery was made by an experimental physicist, while on the other hand, the final "experimental proof" was provided by the two theoretical physicists who analyzed existing data correctly. The moral of this is, of course, that one should not just be either an experimentalist or a theoretician. In the final analysis, the important thing is to be a physicist.

\section{References}

1. Teller, E. and WHEeler, J., Phys. Rev. 53 (1938) 778

2. RAINWATER, J., Phys. Rev. 79 (1950) 432

3. BOHR, A., Phys. Rev. 81 (1950) 134

4. GOLDHABER, M. and SUNYAR, A.W., Phys. Rev. 83 (1951) 986

5. BOHR, A., Dan. Mat. Fys. Medd. 26 (1952) No. 14

6. BOHR, A. and Mottelson, B.R., Phys. Rev. 89 (1953) 316

7. BOHR, A. and Mottelson, B.R., Phys. Rev. 90 (1953) 717

8. BOHR, A. and Mottelson, B.R., Dan. Mat. Fys. Medd. 27 (1953) No. 16

\title{
Physics Education in the German Democratic Republic
}

\section{J. Auth, Berlin}

(Chairman of the National Committee on Physics of the GDR)

Science plays a very important role in a socialist country and consequently, education in science is also a matter of great importance. When the new five-year plan, 1976-1980, was prepared, a long term programme on basic research in mathematics, in the fundamental natural sciences and also in some technical sciences was worked out and adopted by the Central Committee of the Socialist Unity Party of Germany and by the Council of Ministers of the GDR. Many competent physicists took part in the elaboration of this document. It gives a sound basis for the further development of physics in our country. A fundamental idea in the programme is, that basic research should be planned and performed in such a way, that both the internal needs of the sciences to ensure their evolution and, the needs of society are fulfilled at the same time. This may be understood also as a fundamental idea in physics education, underlying the physics education in the schools as well as in the Universities.

\section{Physics Education in the Schools}

The main type of school in the GDR is the "allgemeinbildende polytechnische Oberschule" (general polytechnical school). It has 10 forms, beginning in the 1st. form with pupils from six years old. It gives a general education in German and one or two foreign languages, in mathemetics, natural sciences, in some social sciences and arts, as well as some polytechnical training. Attending the school until the age of 16 is a legal obligation and free of charge, as is all education in our country. Pupils intending to go to university, technical highschools (technological institutes) or to the Technical University of Dresden have to complete their general education with two further years in the 11th. and 12th. form of the "erweiterte polytechnische Oberschule" (extended polytechnical secondary school) at the ages of 17 and 18 years, or by some other form of further education. After thorough examinations in the 12th. form, the successful students receive a certificate, the "Abitur", qualifying them to attend university without any further examination.

Physics education together with mathematics and the other natural sciences is essential to the "Integrated Socialist Educational System", as learning physics makes an important contribution to forming the personality of a human being. Exact and systematic knowledge of natural phenomena, of the principles, rules and laws that underlie them, and the theories that are derived from this knowledge, are of great importance in understanding the world, the objective character of nature and science and for getting deeper insight into the philosophy of dialectical materialism. We agree with Casimir') that physics teaching in school "should lead to an understanding of physics underlying everyday life, and more specifically its technological aspect", and we think also with him, that "if it is done well, such teaching should automatically convey an appreciation of the beauty of physics - and help prospective physicists to make up their minds". We agree also with Weisskopf, that physics should be taught in such a way that everybody understands that it is human.

Education in physics begins in the 6 th. form (12 years of age); it takes $8-10 \%$ of the lessons, that means, as a rule, 3 hours a week. Mathematics takes about $14 \%$, chemistry $6 \%$ and biology $5 \%$. For pupils with a special interest in physics, there is additional time for practical scientific work and for voluntary facultative lessons. The total time devoted to physics from the 6 th. to the 12th. form of 555 lessonhours is distributed amongst the different fields of physics as follows : $31 \%$ mechanics, $25 \%$ electricity, $14 \%$ heat, $6 \%$ optics, $6 \%$ quantum physics, $2 \%$ nuclear physics, $1 \%$ relativity, $7 \%$ general questions and revision, $8 \%$ concentrated laboratory work. Besides the $8 \%$ concentrated 
laboratory work, pupils are making experiments during the lessons, even at the beginning of the 6th. form. Practical experimental work occupies, as a result, much more than $8 \%$ of the time. Generally, the schools are well equipped with instruments for experimental demonstrations by the teacher as well as for pupils' experimental work.

The physics education programme of our schools is structured in a modern way. In the 10th. form, for instance, mechanics and electricity are dealt with together under common headings in two sections, with the titles "oscillations" and "waves". In the 11th. form we have quantum physics considered from the general aspect of wave-particle dualism. The 12th. form is dealing with the concept of fields (gravitation and electromagnetic). Much attention is paid to the application of physics in modern industry.

But the structure poses some problems. Some physicists in our country feel, that in the last two forms, classical physics has not been given sufficient attention. The modern structuring under such headings as indicated above throws light on some very essential aspects of physics, but may not be the best way of instilling a reliable knowledge of facts. Pedagogical research on this problem and on creative learning ${ }^{2}$ ) ils going on. The Academy of Sciences and the Academy of Pedagogical Sciences of the GDR are analysing and discussing the questions.

\section{Physic Education in Universities}

There are seven universities and three "technische Hochschulen" where physics majors are educated. In 1974, there were 2265 students in physics, 516 freshmen were beginning and 644 were finishing their studies with the first academic degree of a "Diplomphysiker" (physics diploma) ${ }^{3}$ ). Physics studies are carried out in these 10 institutions on a unified basis. The programme includes a statement of the principles governing the objectives of the studies, of the aims and the main content of the obligatory courses and the time-table. This programme was worked out in 1972-1973 by the Council for Physics Education according to the demands for a further rise in the standards of education and training. It was discussed with a group of well known physicists of the Academy of Sciences of the GDR and we received valuable advice. According to the programme, five years are necessary to obtain a physics diploma.
The aim of the studies is to develop a physicist of broad utility. He should be able to work successfully in basic research as well as in the application of physics in technology. He should be able to apply physics in a creative way to what might, at first sight, appear to be non-physical problems. He should be able to recognize the essential physical content of a technical problem in industry.

The aim of education and training at university is the highly qualified socialist personality. The physicist should therefore be convinced of the human character of science, he should know about his high responsibility to do his best for society.

Lectures, seminars and training in experimental work and theoretical calculations comprise the main content of the first four years. According to the timetable, we have 30 hours in the week and a total sum of 3780 hours in the period. Nearly a quarter $(23 \%)$ is devoted to social sciences, languages and sport; the remaining $77 \%$ is devoted to physics. Within this $77 \%$ we have the following distribution: $26 \%$ mathematics, $16 \%$ classical physics, $14 \%$ quantum physics, $7 \%$ applied physics, $1,6 \%$ chemistry, $28 \%$ practical training in experimental physics, $1,1 \%$ history of physics and the remaining $6 \%$ for special topics according to the main fields of research at the special university.

The last (fifth) year is almost entirely devoted to research (experimental or theoretical) - the diploma work of the student. The student has to perform a rather independent scientific activity in one of the research teams of the physics department of the university or, in special cases, also in other scientific institutes (for instance the Academy of Science) or in industry. In the fourth and partially also in the third year, the student prepares himself for this work by attending special lectures and by completing a more modest research study ("Forschungsbeleg") in the same or a related field, as a part of the above mentioned practical experimental training. The diploma work should be of a high standard and is discussed in a colloquium at the end of the study. It is the basis for getting the degree of a "Diplomphysiker". Very often the results of the diploma work are published in a scientific journal.

Examinations are held at the end of each year in one or two fields. The main examination in mathematics is at the end of the fifth semester in the middle of the third year. The main examinations in theoretical and experimental physics are at the end of the fourth year and a pass is necessary for being admitted to the diploma work.

We think that there are two main working methods in physics - the theoretical and the experimental. All we know about physics principles and laws is the result of both. The main aim in the education of future physicists, and that means more than education in physics, is to make them understand physics as a living science, make them understand how new knowledge arises and so enable them to take part in the process of winning new knowledge about nature and to use this knowledge to the benefit of human society. Every student therefore, who wishes to become a physicist, must learn both the theore-

\section{Technische Hogeschool Delft}

UNIVERSITY OF TECHNOLOGY DELFT, DEPARTMENT OF APPLIED PHYSICS Applications are invited for the position of :

\section{FULL PROFESSOR OF PHYSICS}

This post will become available on January 1, 1977. A broad experience in the development of modern cryogenic techniques is required, involving the construction of apparatus as well as methods of measurement at very low temperatures, to within the millikelvin region. Candidates should, moreover, have a profound knowledge of the fundamental physics which governs the phenomena in this field.

Besides providing courses on the various aspects of low temperature physics, a contribution to the general physics teaching programme of the department will be expected.

Applications, including a curriculum vitae, an account of professional experience and publications, and the names of two referees should be sent to:

Professor J. B. Westerdijk, Laboratory of Applied Physics,

Lorentzweg 1, Delft, The Netherlands,

where further information concerning the post and the department may be obtained. 
tical and experimental methods. But to teach and to learn the experimental method is difficult. It may be more difficult than the theoretical, that is well formalized, complete etc. In consequence, care must be taken that the experimental method is not underweighted in physics education. We think we have payed attention to this in our programme. Leading the student step by step from the very beginning, with simple experiments in the first year to real research in the last year, we believe efficiently overcomes the difficulties. We think, laboratory work is necessary in every phase of education. That is why we have a sequence of laboratory courses, growing with time in level and aims.

It is very important also, that our students know how physics acts in modern industry, how a new technology arises as a teamwork of scientists and engineers from several different fields. It is necessary to prepare the student for this teamwork. In some lectures, summed up above as applied physics, we try to give the stu- dent some idea of physics in modern industry. We have here about 96 hours measuring techniques (3rd. until 5th. semester), 64 hours electronics (3rd. semester), 32 hours solid state electronics (8th. semester) and 32 hours technology for physicists (8th. semester) with the double aim, to give the students new knowledge for their practical experimental work, but also to show them how physics is used in various fields of technology. Especially in the lectures on technology for physicists, it is shown, using one suitable example (e.g. integrated circuits), how physics is used in the development of a new product, in the different production steps, in production control, and in the application of the product. It is shown how physics, chemistry, mathematics, material science, and several fields of engineering have to act together, to arrive at a stable and efficient production of a modern product, and it is also shown that physics and economics in the production process are not independent of one another.

Very important in this connection is also the "Industrie-praktikum" at the end of the third year. The student is working for three months in an industrial plant in the place of a physicist. $\mathrm{He}$ has to solve a (smaller) problem, as it would be solved by a physicist in the industry. He has to cooperate with other scientists, engineers and technicians, as is typical of teamwork in industry. We think that this work is very important for establishing the right understanding of the part physics plays in industry and for developing an additional motivation for the last two years, especially for the diploma work.

\section{References}

1. CASIMIR, H.B.G., Physics Education 11 (1976) 11.

2. LECHNER H., Wissenschaftliche Konferenz der Forschungsgemeinschaft der Methodikbereiche Biologie, Chemie und Physik. (Humboldt-Universität. Berlin am 17. und 18. März 1976) To be published

3. Statistiches Jahrbuch der DDR 1975 (Staatsverlag der DDR, Berlin) 1975, p. 344.

\title{
Microprocessor Applications in Physics
}

\author{
T.F. Buckley, E.R.S. Candlin, D.J. Candlin and A. Q. Jones, Edinburgh Univ. \\ (Computer Science Department) \\ (Physics Department)
}

Why are so many conferences and articles in journals devoted to microprocessors these days? Do they represent some new breakthrough in the computer field? Are they of any use in experimental physics? What is a microprocessor anyway?

We should like to suggest answers to some of these questions, and to show that microprocessors do indeed have a significant role to play, both in the control of equipment and as components in very fast scientific computers. We are currently building a fast "number-cruncher" ourselves, and we present this as an example, to illustrate some of the points which have to be considered when designing a microprocessor system.

\section{How microprocessors work}

Microprocessors are very large scale integrated circuits which, on a single chip, have an arithmetic and logic unit, a set of registers, and a control section, linked internally by networks of greater or lesser complexity (Fig. 1). Each microprocessor can perform any of a set of basic operations (e.g. transfer, add) taking its operands from internal registers or from its input. Microprocessor designs differ widely in speed, word length, operation set and price, but all execute a series of microinstructions in a microroutine in response to a user instruction. We can distinguish two general classes of microprocessor. The first carries a set of microinstructions in a Read-Only control memory, integral with the chip. The manufacturer fixes the available set of (user) instructions when he designs the control microprogram. The second type requires an external microprogram memory. This is provided by the user who can, therefore, define his own set of instructions in terms of microroutines. The programmability of this second type makes them particularly powerful and flexible. Those interested in learning about microprocessors may find Refs. 1-6 helpful.

\section{Useful tool for physicists}

We can distinguish two rather different ways in which physicists employ computers. On the one hand, there is large-scale scientific data processing carried out by, for exam- ple, high energy physicists, meteorologists and crystallographers. These people usually make use of large main frame computers. They are generally competent high level language programmers, but are probably not interested in the lower-level hardware of the machine they are using nor in the details of its operating system. On the other hand, there are physicists who require real-time control of experimental apparatus. For this purpose they use minicomputers, normally dedicated to the specific task.

If we now look at the current commercial use of microprocessors, we can again see two rather different types of application. In the first case, there is the "petrol pump" application, i.e. using the microprocessor as a dedicated controller for a piece of apparatus. In the second case, microprocessors may form the basis of a more complicated piece of computing machinery, for example, the central processing unit of a minicomputer.

These uses are clearly parallel to the requirements of the two broad categories of physics use, and physicists may sometimes be able to buy what 